\title{
The Importance of Prognostic Nutritional Index in Predicting Acute Renal Failure After On-Pump Coronary Artery Bypass Operations in Patients with Insulin-Dependent Diabetes Mellitus
}

\author{
Arif Gucu, MD,${ }^{1}$ Ozlem Arican Ozluk, MD, ${ }^{2}$ Orhan Guvenc, MD, ${ }^{3}$ Sadik Ahmet Sunbul, MD, ${ }^{1}$ \\ Mesut Engin, $\mathrm{MD}^{1}$ \\ ${ }^{1}$ University of Health Sciences, Bursa Yuksek Ihtisas Training and Research Hospital, Department of Cardiovascular Surgery, Yıldırım/ \\ BURSA, Turkey; ${ }^{2}$ University of Health Sciences, Bursa Yuksek Ihtisas Training and Research Hospital, Department of Cardiology, BURSA, \\ Turkey; ${ }^{3}$ Medical Faculty of Uludağ University, Departments of Cardiovascular Surgery, Bursa, Turkey
}

\section{ABSTRACT}

Background: After coronary artery bypass graft (CABG) operations, acute kidney injury (AKI) appears at 5-30\% rates, and this rate increases even more in patients with diabetes mellitus (DM). Prognostic nutritional index (PNI) is known as a valuable parameter that affects cardiovascular surgery outcomes. In this current study, we aimed to investigate the importance of PNI value in predicting AKI after on-pump CABG operations in insulin-dependent diabetic patients.

Methods: A total of 254 consecutive patients with insulindependent diabetes who underwent on-pump CABG in our clinic between January 2016 and January 2020 retrospectively were included in this study. In the postoperative period, patients were registered as the renal failure group (Group 1), and those who did not develop renal failure were registered as Group 2.

Results: A total of 255 patients with DM were included in the study. There were 82 patients in Group 1 and 173 patients in Group 2. There was no difference between the groups, in terms of age, gender, smoking, and hyperlipidemia rates. Hypertension rate significantly was higher in Group 2 $(P=.001)$. In multivariate logistic regression analysis, hypertension (OR: 1.226, 95\% CI: $1.114-2.459, P=.026$ ), need for inotropic support (OR: 1.128, 95\% CI: 1.070-1.784, $P$ $=.033)$, increased blood product use (OR: 1.291, 95\% CI: $1.112-2.156, P=.021)$ preoperative high creatinine (OR: 3.563, 95\% CI: 2.497-5.559, $P<.001$ ), and PNI (OR: 1.327 , $95 \%$ CI: $1.118-2.785, P=.012)$ were independent predictors of AKI.

Conclusion: In our study, we determined PNI value as an independent predictor in predicting acute renal injury occurring after on-pump CABG operations in patients with insulin-dependent DM.

Received April 1, 2021; accepted May 3, 2021.

Correspondence: Arif Gucu, MD, Assoc Prof, University of Health Sciences, Bursa Yuksek Ibtisas Training and Research Hospital, Department of Cardiovascular Surgery, Mimar Sinan Town, Emniyet Street, Yildirn/ BURSA, Turkey; +90224-295-50-00; fax: +90224-275-67-67 (e-mail: dr.arifgucu@gmail.com).

\section{INTRODUCTION}

Today, coronary artery bypass graft (CABG) operations are widely performed, and the success of the procedure is influenced by complications that develop after the operation besides surgical technique [Erdolu 2020]. One of the most important complications is acute kidney injury (AKI) clinic, which can occur after the operations [Usta 2021]. In addition, $20-30 \%$ of diabetes mellitus (DM) is detected in patients with CABG planned. After these operations, AKI appears at a rate of $5-30 \%$, and this rate increases even more in patients with DM [Kubal 2005].

Common risk factors affecting AKI development after CABG operations are parameters, such as advanced age, hypertension (HT), and prolonged perfusion periods [Gaffney 2015]. Recently, the prognostic value of the prognostic nutritional index (PNI), in which the effects of cardiovascular surgery on clinical outcomes are investigated, has become an important parameter. This value is calculated by a formula that consists of albumin and lymphocyte values. In a recent study, it has been shown to be an important parameter in predicting early clinical outcomes after adult cardiac surgery [Lee 2020]. In another study, it was revealed that it can predict the development of AKI in the postoperative period in patients undergoing on-pump CABG [Dolapoglu 2019].

In this current study, we aimed to investigate the importance of PNI value in predicting AKI that occurs after on-pump CABG operations in insulin-dependent diabetic patients.

\section{MATERIALS AND METHODS}

A total of 254 consecutive patients with insulindependent diabetes who underwent on-pump CABG in our clinic between January 2016 and January 2020 retrospectively were included in this study. The study was initiated after the approval of the local ethics committee. The study was carried out in accordance with the Helsinki Declaration criteria. Demographic data of all patients (age, gender, body mass index, etc.), preoperative blood values (hemoglobin, white blood cell, lymphocyte, albumin, C-reactive protein, etc.), 


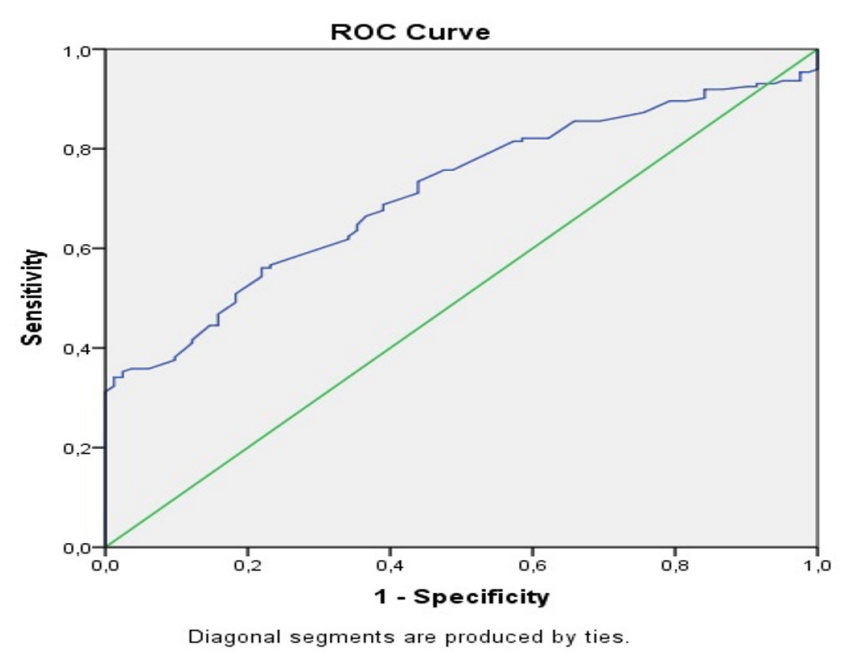

Data figure of the area under the curve (AUC), confidence interval (CI), and cut-off values in receiver-operating characteristic (ROC) curve analysis for prognostic nutritional index (PNI) to predict acute kidney injury (cut off: 42.9, AUC: 0.711, 95\% Cl:0.649- 0.774, $P<.001$, with $56.6 \%$ sensitivity and $76.8 \%$ specificity).

operative (cardiopulmonary bypass and aortic cross-clamping (ACC) duration), and postoperative features (hospitalization and critical care periods, blood product usage status, etc.) were accessed from the hospital-based registry and patient follow-up cards, and the data were recorded.

Those with concomitant cardiac surgical disease, serum creatinine level above $1.5 \mathrm{mg} / \mathrm{l}$ in the preoperative period, liver failure, active infection or malignancy, preoperative hemoglobin level of $10 \mathrm{~g} / \mathrm{dl}$ and below, and patients with congestive heart failure were excluded from the study. All patients included in the study had insulin-dependent DM.

Blood parameter analysis: Evaluations of blood parameters of all patients were made from blood samples taken from preoperative antecubital veins. Preoperatively, the PNI value was calculated using the following formula [Onodera 1984].

$\mathrm{PNI}=$ serum albumin levels $(\mathrm{g} / \mathrm{dl}) \times 10+$ total lymphocyte count $\left(\right.$ per $\left.\mathrm{mm}^{3}\right) \times 0.005$

Surgical technique: All operations were performed under general anesthesia with a median sternotomy. Standard CPB was used in mild hypothermia $\left(32^{\circ} \mathrm{C}\right)$ with aorta-venous two-stage cannulation. Cardioplegic arrest was achieved. Cardiopulmonary bypass was provided by roller pumps at a flow rate of 2-2.4 L/ $\mathrm{min} / \mathrm{m}^{2}$ and a membrane oxygenator (Maquet, Getinge Group, Rastatt, Germany). Arterial filters were used in all operations. All distal anastomoses were performed under ACC, while proximal anastomoses were performed using the partial clamp technique. All patients received warm potassium-free blood cardioplegia before the ACC was removed. All patients were transferred to the cardiovascular surgery intensive care unit after the operation.

Diagnosis of postoperative acute kidney injury: In the postoperative period, blood parameters of the patients were monitored for two days, and AKI was determined according to creatinine changes [Mehta 2007]. Postoperative AKI was made, according to the Kidney Disease Improving Global
Table 1. Demographic data, preoperative features and of the patients

\begin{tabular}{lccc}
\hline Variables & Group 1 (N=82) & Group 2(N=173) & $P$ \\
\hline Age (years) & $62.7 \pm 9.8$ & $63.9 \pm 8.7$ & $.174 \dagger$ \\
Male gender, n (\%) & $50(60.9 \%)$ & $101(58.3 \%)$ & $.694^{*}$ \\
BMl (kg/m²) & $29.5(23-35.4)$ & $29.2(22-36)$ & $.457 \ddagger$ \\
Hypertension, n (\%) & $53(64.6 \%)$ & $72(41.6 \%)$ & $.001 *$ \\
COPD, n (\%) & $17(20.7 \%)$ & $33(19 \%)$ & $.756^{*}$ \\
Previous PCl, n (\%) & $32(39 \%)$ & $58(33.5 \%)$ & $.391 *$ \\
Smoking, n (\%) & $18(21.9 \%)$ & $35(20.2 \%)$ & $.752 *$ \\
Hyperlipidemia, n (\%) & $44(53.7 \%)$ & $90(52 \%)$ & $.807 *$ \\
Ejection fraction (\%) & $50(35-65)$ & $50(35-65)$ & $.634^{*}$ \\
ASA use, n (\%) & $60(73.1 \%)$ & $133(76.8 \%)$ & $.578 *$ \\
ACEl/ARB use, n (\%) & $65(79.2 \%)$ & $145(83.8 \%)$ & $.417 *$ \\
\hline
\end{tabular}

* Chi-square test; $\dagger$ Student’s t-test; $\ddagger$ Mann-Whitney $U$ test ACEl, angiotensin-converting enzyme inhibitor; ARB, angiotensin receptor blocker; ASA, acetylsalicylic acid; BMI, body mass index; COPD, chronic obstructive pulmonary disease; $\mathrm{PCl}$, percutaneous coronary intervention

Outcomes (KDIGO) classification [Tumet 2019].

This classification is made under three headings:

Stage 1: $1.5-1.9$ times, or $>0.3 \mathrm{mg} / \mathrm{dl}$ increase $>$ from the basal rate

Stage 2: 2.0-2.9 times increase from basal rate

Stage 3: 3 times increase from basal rate or Serum creatinine $>4.0 \mathrm{mg} / \mathrm{dl}$ or initiation of renal replacement therapy

According to the results of this evaluation, patients who developed any of these stages in the postoperative period were registered as the renal failure group (Group 1), and those who did not were registered as Group 2.

Statistical analysis: Statistical analysis was performed with SPSS 21.0 (IBM Statistical Package for the Social Sciences Statistic Inc. version 21.0, Chicago, IL, USA). Ordinal and continuous data were expressed in mean and standard deviation. Normality distribution of data was assessed with KolmogorovSmirnov and Shapiro-Wilk tests. Student's t-test was used for normally distributed data (data expressed as mean, standard deviation), and Mann Whitney U test was used for non-normally distributed data (data expressed as mean, interquartile range). Nominal variables were expressed in frequency and percentage, and Chi-Square test was used for analysis. AKI predictors were evaluated with binary logistic regression analysis. The predictive value of PNI was determined with Receiver Operating Characteristic (ROC) curve analysis and calculation of Area Under Curve (AUC). A $P$ value <.05 was considered significant.

\section{RESULTS}

A total of 255 patients with DM were included in the study. There were 82 patients in Group 1 (with renal injury) 
Table 2. Preoperative laboratory variables of the patients

\begin{tabular}{lccc}
\hline Variables & $\begin{array}{c}\text { Group 1 } \\
(\mathrm{N}=82)\end{array}$ & $\begin{array}{c}\text { Group 2 } \\
(\mathrm{N}=173)\end{array}$ & Pf \\
\hline White blood cell $\left(10^{3} / \mu \mathrm{L}\right)$ & $8.2(6.6-10.1)$ & $8.4(6.3-10.4)$ & .236 \\
Hemoglobin $(\mathrm{mg} / \mathrm{dl})$ & $12.7(11.6-14)$ & $13.1(10.9-13.7)$ & .118 \\
Platelet $\left(10^{3} / \mu \mathrm{L}\right)$ & $244(158-355)$ & $238(139-430)$ & .214 \\
Neutrophil $\left(10^{3} / \mathrm{\mu L}\right)$ & $4.8(2-7.8)$ & $4.6(2-9.6)$ & .096 \\
Lymphocyte $\left(10^{3} / \mu \mathrm{L}\right)$ & $1.8(0.8-3.3)$ & $2(0.7-4.2)$ & .010 \\
Creatinine, $\mathrm{mg} / \mathrm{dL}$ & $1.3(0.6-1.46)$ & $0.9(0.8-1.38)$ & $<.001$ \\
Albumin $(\mathrm{g} / \mathrm{L})$ & $36.9(34.7-50)$ & $38.1(35-52)$ & $<.001$ \\
HbA1c $(\mathrm{mmol} / \mathrm{mol})$ & $8.9(7.7-13.4)$ & $8.3(7.9-12.1)$ & .015 \\
CRP $(\mathrm{mg} / \mathrm{dL})$ & $8.6(1.8-21.7)$ & $8.4(2-32.4)$ & .356 \\
PNI & $43(35-59)$ & $44.7(36-63)$ & $<.001$ \\
\hline
\end{tabular}

$\ddagger$ Mann-Whitney $U$ test.

HbA1c, hemoglobin A1c; CRP, C-reactive protein; PNI, prognostic nutritional index

and 173 patients in Group 2 (without renal injury). There was no difference between the groups, in terms of age, gender, BMI, COPD, previous PCI, smoking, and hyperlipidemia rates. Hypertension rate significantly was higher in Group 2 $(P=.001)$. Preoperative EF and use of angiotensin-converting enzyme inhibitor, angiotensin receptor blocker, and acetylsalicylic acid were similar in both groups. Preoperative demographic data of the patients are shown in Table 1.

Preoperative blood parameters of all patients are given in Table 2. There was no difference between the groups, in terms of hemoglobin, platelet counts, neutrophil, and $\mathrm{C}$-reactive protein value. Creatinine and $\mathrm{HbA1c}$ values were significantly higher in Group $1(P<.001$ and $P=.015$, respectively). And lymphocyte, albumin, and PNI values were significantly lower in Group $1(P=.010, P<.001$, and $P<.001$, respectively).

Operative and postoperative characteristics of the patients are shown in Table 3. The two groups were similar, in terms of cross-clamp time and number of distal anastomoses. Total perfusion time, total amount of blood product used, need of inotropic support, total hospital stays, and total intensive care stays were significantly higher in Group $2(P=.012, P<.001$, $P=.004, P<.001$, and $P<.001$, respectively).

To analyze the factors affecting the development of AKI in the postoperative period, univariate and multivariate logistic regression analysis was performed (Table 4). In univariate analysis, hypertension (OR [odds ratio]: 1.645, 95\% CI [confidence interval]: $1.315-2.678, P=.002)$, total perfusion time (OR: 1.514, 95\% CI: $1.116-1.994, P=.015)$, need for inotropic support (OR: 1.442, 95\% CI: $1.176-2.655, P=.006)$, increased blood product use (OR: 2.114 , 95\% CI: $1.877-$ $2.945, P<.001$ ), preoperative high creatinine (OR: 4.114, 95\% CI: 2.664-6.312, $P<.001)$, low lymphocyte count (OR: 0.889, 95\% CI: $0.558-0.992, P=.012$ ), low albumin (OR:
Table 3. Operative and postoperative features of the patients

\begin{tabular}{lccc}
\hline Variables & $\begin{array}{c}\text { Group 1 } \\
(\mathrm{N}=82)\end{array}$ & $\begin{array}{c}\text { Group 2 } \\
(\mathrm{N}=173)\end{array}$ & $P$ \\
\hline Total perfusion time & $104(66-170)$ & $102(68-158)$ & $.012 \ddagger$ \\
Cross-clamp time & $78(28-90)$ & $75(30-101)$ & $.128 \ddagger$ \\
Number of distal anastomoses & $4(3-6)$ & $4(3-6)$ & $.114 \ddagger$ \\
Packed blood products (units) & $6(4-12)$ & $5(4-9)$ & $<.001 \ddagger$ \\
Inotropic support, $\mathrm{n}(\%)$ & $27(32.9 \%)$ & $29(16.7 \%)$ & $.004^{*}$ \\
Total ICU stay (days) & $4(2-21)$ & $2(2-12)$ & $<.001 \ddagger$ \\
Total hospital stay (days) & $8(7-35)$ & $7(6-21)$ & $<.001 \ddagger$ \\
\hline
\end{tabular}

* Chi-square test; $\ddagger$ Mann-Whitney $U$ test

1.978, 95\% CI: 1.334-2.323, $P<.001)$, high HbA1c (OR: 1.114, 95\% CI: $1.090-1.345, P=.019$ ), and low PNI (OR: 2.428, 95\% CI: $1.678-3.994, P<.001)$ were correlated with the development of AKI. In multivariate logistic regression analysis, hypertension (OR: 1.226, 95\% CI: 1.114-2.459, $P=$ $.026)$, need for inotropic support (OR: 1.128, 95\% CI: $1.070-$ $1.784, P=.033$ ), increased blood product use (OR: 1.291, 95\% CI: $1.112-2.156, P=.021)$ preoperative high creatinine (OR: 3.563, 95\% CI: 2.497-5.559, $P<.001$ ), and PNI (OR: 1.327, 95\% CI: $1.118-2.785, P=.012)$ were independent predictors of AKI.

ROC analysis was performed to evaluate cardioplegia volume in predicting AKI after CABG operations performed with cardiopulmonary bypass. The cut-off value was 42.9 (area under the curve: 0.711 , confidence interval: 0.649 $0.774, P<.001$, with $66.6 \%$ sensitivity and $76.8 \%$ specificity).

\section{DISCUSSION}

After coronary bypass operations, AKI is seen at a considerable rate, and this rate increases even more in DM patients. This prolongs the length of stay and increases the cost of treatment. In this current study, we first found the PNI value calculated preoperatively in patients with insulin-dependent $\mathrm{DM}$ as an independent predictor in predicting the development of AKI.

Malnutrition is an important health problem, especially in developing countries. PNI, which is obtained from serum albumin value and lymphocyte values, is a valuable parameter used as an indicator of malnutrition. Serum albumin plays an important role in providing plasma oncotic pressure and is an important acute phase reactant. It also has anti-inflammatory and antioxidant effects [Arques 2018]. Studies have shown that hypoalbuminemia is associated with postoperative mortality and morbidity [de la Cruz 2011]. Lymphocytes play an important role in the development and prognosis of cardiovascular diseases. Neutrophil count increases as the cellular immune system is activated by lymphocytes. Due to this mechanism, the NLR increases, and 
Table 4. Logistic regression analysis to identify factors affecting postoperative acute kidney injury

\begin{tabular}{|c|c|c|c|c|c|c|}
\hline Hypertension & .002 & 1.645 & $1.315-2.678$ & .026 & 1.226 & $1.114-2.459$ \\
\hline Total perfusion time & .015 & 1.514 & $1.116-1.994$ & .334 & 0.886 & $0.578-1.120$ \\
\hline Inotropic support & .006 & 1.442 & $1.176-2.655$ & .033 & 1.128 & $1.070-1.784$ \\
\hline Blood product use & $<.001$ & 2.114 & $1.877-2.945$ & .021 & 1.291 & $1.112-2.156$ \\
\hline Pre-creatinin & $<.001$ & 4.114 & $2.664-6.312$ & $<.001$ & 3.563 & $2.497-5.549$ \\
\hline Lymphocyte count & .012 & 0.889 & $0.558-0.992$ & - & - & - \\
\hline Albumin & $<.001$ & 1.978 & $1.344-2.323$ & - & - & - \\
\hline
\end{tabular}

HbA1c, hemoglobin A1c; PNI, prognostic nutritional index

this rate takes an important place with many cardiovascular diseases [Abanoz 2021]. The lymphocyte count, which also affects the PNI value as $x 5$, significantly affects this value.

There are various studies in the literature investigating the effect of PNI value on clinical outcomes after cardiac operations. In a study conducted by Lee et al, the prognostic role of PNI value in predicting early clinical outcomes after cardiac surgery was investigated. In this study, the authors divided the patients into two groups, according to the value they determined as cut-off (46.13). At the end of the study, early mortality rates were found to be higher in the group with low PNI values. In addition, the mean length of stay on mechanical ventilation and length of stay in the intensive care unit were found to be significantly higher in this group [Lee 2020]. In another study by Hayashi et al., low PNI was shown as an independent predictor of mortality after cardiac operations (hazard ratio $0.40 ; 95 \%$ confidence interval $0.18-0.90, P=$ .026) [Hayashi 2020].

In a study conducted by Dolapoglu et al, similar to our study, the role of PNI value in predicting AKI in patients who underwent CABG was investigated. A total of 336 (35.1\% with DM) patients were included in the study, and the rate of AKI was found to be $26.2 \%$. The authors found that PNI was correlated with postoperative AKI [Dolapoglu 2019]. In our study, we determined the PNI value as an independent predictor for AKI. However, unlike this study, our study consisted of patients with insulin-dependent diabetes, so the rate of AKI also was higher in our study group (32.1\%). In a study conducted by Aksoy et al, the role of low albumin in patients with DM in predicting AKI that develops after CABG operations was investigated. Six hundred thirty-four patients were included in the study, and $36.2 \%$ of the patients developed AKI. The authors showed hypoalbuminemia as an independent predictor for AKI (OR and 95\% CI, 0.453 [0.216-0.947]; $P=.035)$. In our study, the albumin used in the calculation of PNI value was significantly lower in the patient group who developed AKI. However, our study stands out from this study because it is in insulin-dependent diabetic patients and included the HbAlc values of the patients. Because in the study by Aksoy et al, DM was defined as the use of any diabetic drug or blood glucose over 126mg/dl [Aksoy 2019]. However, long-term regulation of blood glucose in patients with DM affects the complications associated with the disease [Nalysnyk 2010].

Renal, retinal damage or peripheral vasculopathies may occur due to microvascular complications in diabetic patients. In a study conducted by Yang et al, the effect of PNI value on the development of diabetic retinopathy was investigated. The authors included $1023 \mathrm{DM}$ patients in the study. As a result of the study, they found that low PNI values were an independent predictor of retinopathy, its presence and severity in patients with DM [Yang 2021]. In our current study, we determined the PNI value in patients with insulin-dependent diabetes as an independent predictor of postoperative AKI. Blood sugar control also is an important condition for microvascular complications. Accordingly, HbA1c values also were found to correlate with the development of AKI in our study (OR: $1.114, P=.019)$. In one study, even in nondiabetic patients, preoperative high AKI values were found to be associated with increased AKI rate after CABG operations [Kocoglulları 2017]. In CABG operations performed with cardiopulmonary bypass, systemic inflammatory response syndrome occurs, due to the contact of blood with foreign surfaces. As a result, immune system and vascular endothelial activation develops and renal tubule injury occurs due to secreted proinflammatory cytokines and free radicals. Neutrophils, macrophages, and lymphocytes activated by cytokines cause renal parenchymal injury [Asimakopoulos 1998]. Studies have found that prolonged $\mathrm{CPB}$ durations are associated with AKI. In our study, we 
determined the increased $\mathrm{CPB}$ duration as an independent predictor for AKI.

Increased blood product usage and positive inotropic support also are important factors that can trigger the development of AKI after CABG operations. As a result of increased hemolysis due to the use of blood products, renal injury may occur due to hemolysis-derived products [Freeland 2015]. Inotropic support may cause renal damage due to vasoconstrictive effects [Chen 2021]. In our study, we identified increased blood product and inotropic support use as independent predictors in predicting the development of AKI.

The most important limitation of our study is that it is a retrospective single-center study. The number of patients was limited as it included patients with insulin-dependent DM. In addition, due to its retrospective nature, it was not possible to study valuable markers, such as cystatin C, neutrophil gelatinase associated lipocalin, and kidney injury molecule 1 [Yuan 2019].

\section{CONCLUSION}

Diabetes mellitus is a cause that leads individuals to coronary bypass operation by promoting atherosclerosis, and it can also affect postoperative clinical results. It is a known fact that patients with DM are more susceptible to postoperative complications than patients without DM. In conclusion, in our study, we determined PNI value as an independent predictor in predicting acute renal injury occurring after onpump CABG operations in patients with insulin dependent DM. Our study includes diabetic patients, which is a special patient group, and it is very important to reveal the risk factors for this patient group, which is quite susceptible to postoperative complications. There is a need for new multi-center prospective studies in this area.

\section{REFERENCES}

Abanoz M, Engin M. 2021. The effect of the relationship between postcardiotomy neutrophil/lymphocyte ratio and platelet counts on early major adverse events after isolated coronary artery bypass grafting. Turk Gogus Kalp Damar Cerrahisi Derg. 29(1):36-44.

Aksoy R, Adademir T, Yilmaz E, et al. 2019. Is Hypoalbuminemia a Predictor for Acute Kidney Injury after Coronary Bypass Grafting in Diabetes Mellitus Patients? Braz J Cardiovasc Surg. 34(5):565-571.

Arques S. 2018. Human serum albumin in cardiovascular diseases. Eur J InternMed. 52:8-12.

Asimakopoulos G, Taylor KM. 1998. Effects of cardiopulmonary bypass on leukocyte and endothelial adhesion molecules. Ann Thorac Surg. 66: 2135-44.

Chen WC, Lin MH, Chen CL, et al. 2021. Comprehensive Comparisons among Inotropic Agents on Mortality and Risk of Renal Dysfunction in Patients Who Underwent Cardiac Surgery: A Network Meta-Analysis of
Randomized Controlled Trials. J Clin Med. 10:1032.

de la Cruz KI, Bakaeen FG, Wang XL, et al. 2011. Hypoalbuminemia and long-term survival after coronary artery bypass: a propensity score analysis. Ann Thorac Surg. 91(3):671-5.

Dolapoglu A, Avci E, Kiris T, Bugra O. 2019. The predictive value of the prognostic nutritional index for postoperative acute kidney injury in patients undergoing on-pump coronary bypass surgery. J Cardiothorac Surg. 14(1):74.

Erdolu B, As AK, Engin M. 2020. The Relationship between the HATCH Score, Neutrophil to Lymphocyte Ratio and Postoperative Atrial Fibrillation After Off-Pump Coronary Artery Bypass Graf Surgery. Heart Surg Forum. 23(1):E88-E92.

Freeland K, Hamidian Jahromi A, Duvall LM, et al. 2015. Postoperative blood transfusion is an independent predictor of acute kidney injury in cardiac surgery patients. J Nephropathol. 4(4):121-6.

Gaffney AM, Sladen RN. 2015. Acute kidney injury in cardiac surgery. Curr Opin Anaesthesiol. 28(1):50-9.

Hayashi J, Uchida T, Ri S, et al. 2020. Clinical significance of the prognostic nutritional index in patients undergoing cardiovascular surgery. Gen Thorac Cardiovasc Surg. 68(8):774-779.

Kocogulları CU, Kunt AT, Aksoy R, et al. 2017. Hemoglobin A1c Levels Predicts Acute Kidney Injury after Coronary Artery Bypass Surgery in Non-Diabetic Patients. Braz J Cardiovasc Surg. 32(2):83-89.

Kubal C, Srinivasan A K, Grayson AD, et al. 2005. Effect of risk-adjusted diabetes on mortality and morbidity after coronary artery bypass surgery. Ann Thorac Surg. 79(5):1570-6.

Lee SI, Ko KP, Choi CH, et al. 2020. Does the prognostic nutritional index have a predictive role in the outcomes of adult cardiac surgery? J Thorac Cardiovasc Surg. 160(1):145-153.e3.

Mehta RL, Kellum JA, Shah SV, et al. 2007. Acute kidney injury network. Acute kidney injury network: report of an initiative to improve outcomes in acute kidney injury. Crit Care. 11(2):R31.

Nalysnyk L, Hernandez-Medina M, Krishnarajah G. 2010. Glycaemic variability and complications in patients with diabetes mellitus: evidence from a systematic review of the literature. Diabetes Obes Metab. 12(4):288-98.

Onodera T, Goseki N, Kosaki G. 1984. Prognostic nutritional index in gastrointestinal surgery of malnourished cancer patients. Nihon Geka Gakkaizasshi. 85(9): 1001-5.

Tumer NB, Kunt AT, Gunaydin S. 2019. Preoperative Urinary pH is Associated with Acute Kidney Injury Afer Cardiac Surgery in Non-Diabetic Patients. Heart Surg Forum. 22(5):E456-E461.

Usta S, Abanoz M. 2021. Can Preoperative Neutrophil To Lymphocyte Ratio Change (Deltanlr) Be Used as a Parameter in Predicting Acute Renal Failure Following Coronary Bypass Operations With Cardiopulmonary Bypass? Heart Surg Forum. 24(1):E194-E200.

Yang L, Yu W, Pan W, et al. 2021. A Clinical Epidemiological Analysis of Prognostic Nutritional Index Associated with Diabetic Retinopathy. Diabetes Metab Syndr Obes. 14:839-846.

Yuan SM. 2019. Acute Kidney Injury after Cardiac Surgery: Risk Factors and Novel Biomarkers. Braz J Cardiovasc Surg. 34(3):352-360. 